\title{
Stationary Tripartite Light-Mirror Entanglement from a Ring Cavity Driven by a Squeezed Light
}

\author{
JUAN GUO ${ }^{a, b, c}$, GUO QUAN YAnG ${ }^{a, c}$ AND Su-Ying Zhang ${ }^{a, c, *}$ \\ ${ }^{a}$ Institute of Theoretical Physics, Shanxi University, Taiyuan 030006, China \\ ${ }^{b}$ College of Physics and Electronics Engineering, Shanxi University, Taiyuan 030006, China \\ ${ }^{c}$ State Key Laboratory of Quantum Optics and Quantum Optics Devices, Shanxi University, Taiyuan 030006, China \\ (Received December 4, 2018; revised version February 12, 2019; in final form March 7, 2019) \\ We study a scheme for generating continuous variable tripartite entanglement of two mirrors and the reflecting \\ field of a ring cavity. We find that significant entanglement can be generated with shot-noise-limited driving field, \\ and the squeezing property of the driving field can enhance the entanglement. We also show the entanglement is \\ generated over a wide range of analysis frequency and temperatures of the environment, which is experimentally \\ accessible. This system may be potentially useful for quantum information processing and quantum networks.
}

DOI: 10.12693/APhysPolA.136.141

PACS/topics: 03.67.-a, 42.50.-p, 42.50.Lc, 42.65.Yj

\section{Introduction}

Multipartite entanglement plays a pivotal role in studying quantum phenomenon from both fundamental [1] and practical $[2,3]$ point of view. On the practical side, efficient generation and manipulation of quantum multipartite entanglement becomes a basic requirement for future quantum network [3]. Especially optical continuous-variable (CV) multipartite entanglement [413], which is described by variables with continuous spectrum, has advantage of efficient manipulation and detection. It has become a valuable resources in protocols such as controlled quantum teleportation [14] and dense coding [15], quantum secret sharing [16], quantum computation [17, 18]. Quantum entanglement between light connects different nodes in the future quantum network. However, taking light as carrier, it is not easy to store quantum information, which is another requirement in the quantum network [3]. Therefore entangling light with other media like mechanical resonators is valuable.

On the fundamental side, quantum correlations including quantum entanglement is crucial in exploring the many fundamental questions of quantum mechanics [1923]. While bipartite quantum correlations seem to be well understood, entanglement of three or more subsystems is still under intensive exploration. Moreover, entanglement of macroscopic objects stands on the border and helps more in deeper understanding of the laws of quantum mechanics.

Cavity optomechanics provides an excellent platform of observing macroscopic quantum effects and holds promising for storing quantum information in quantum networks. Based on this system, various schemes of generating CV bipartite entanglement and tripartite entanglement have been proposed. By feeding a beam of laser to

*corresponding author; e-mail: zhangsy@sxu.edu.cn optomechanical cavity, the optical state can be either entangled with the mechanical resonator [24-27], or transferred to the mechanical resonator [28]. One laser connecting two mechanical resonators can entangle them together, no matter whether the two mechanical resonators are in one cavity [29-32] or two cavities [33-35]. Tripartite entanglement between two optical modes and one cavity mirror can also be generated in this way [36-40]. Here in this paper we also propose to generate tripartite entanglement but between one optical mode and two cavity mirrors. Similar to Refs. [30,31], in our scheme the entanglement also can be enhanced by injecting squeezed light.

Our paper is organized as follows. In Sect. 2, we show the model and give a derivation of the equations of motion according to the Hamiltonian of the system. In Sect. 3, the steady state of the system is studied. In Sect. 4, tripartite entanglement preparation is studied, containing the discussion on the sufficient inseparability criteria and the optimal condition to obtain the maximal entanglement. In Sect. 5, we give a summary,

\section{Theoretical model and equations of motion}

We consider a three-mirror optomechanical cavity, which is driven by amplitude squeezed light through a fixed and partial transmitting mirror. (cf. Fig. 1). Each of the other two mirrors $\mathrm{M}_{1}$ and $\mathrm{M}_{2}$ has perfect reflectance, and is oscillating as a high quality mechanical resonator. In the vicinity of cavity resonance, the injected laser exerts radiation pressure force on each of the two movable mirrors and induces its motion, which changes cavity length and accordingly the intensity of the cavity field. In turn, the radiation pressure force is modified. So the interaction of the injected squeezed light with the movable mirrors through the radiation pressure is a delayed feedback loop and therefore is a nonlinear effect which causes correlations among the cavity mode and the two mirrors' mechanical modes. 


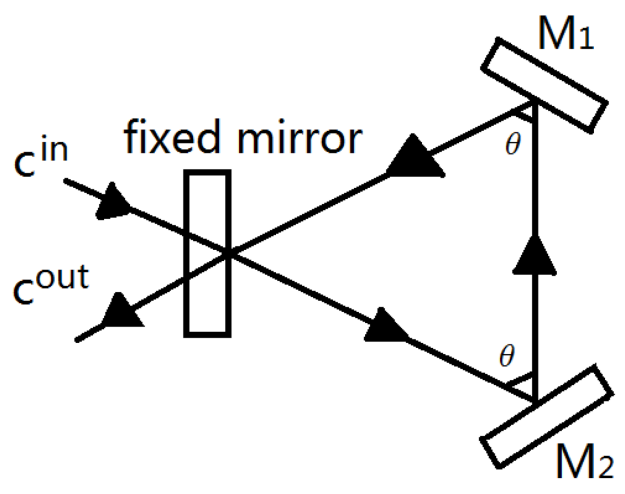

Fig. 1. The ring cavity set up. $\mathrm{M}_{1}$ and $\mathrm{M}_{2}$ are movable mirrors. Field $c^{\text {in }}$ with frequency $\omega_{L}$ is injected into the ring cavity through the fixed mirror.

Assuming that the two movable mirrors are identical with the same mass of $m$, mechanical frequency of $\omega_{m}$, and damping rate of $\gamma_{m}$, free Hamiltonian of the mirrors is given by

$$
\hat{H}_{m}=\frac{1}{2} \hbar \omega_{m}\left(\hat{a}^{+} \hat{a}+\frac{1}{2}\right)+\frac{1}{2} \hbar \omega_{m}\left(\hat{b}^{+} \hat{b}+\frac{1}{2}\right),
$$

where $\hat{a}(\hat{b})$ and $\hat{a}^{+}\left(\hat{b}^{+}\right)$are annihilation operator and creation operator of the mirror $\mathrm{M}_{1}\left(\mathrm{M}_{2}\right)$, respectively.

Supposing that the radiation pressure forces exerted by intracavity field on the mirrors $\mathrm{M}_{1}$ and $\mathrm{M}_{2}$ are opposite [32] and taking into account the angle $\theta$ between the incident light and the reflected light at the surfaces of the movable mirrors, the total Hamiltonian of the system resulting from radiation pressure can be written as

$$
\begin{aligned}
\hat{H} & =\hbar\left(\omega_{c}-\omega_{L}\right) \hat{c}^{+} \hat{c}+\frac{1}{2} \hbar \omega_{m}\left(\hat{a}^{+} \hat{a}+\frac{1}{2}\right) \\
& +\frac{1}{2} \hbar \omega_{m}\left(\hat{b}^{+} \hat{b}+\frac{1}{2}\right)+\frac{1}{\sqrt{2}} \hbar g \hat{c}^{+} \hat{c} \cos ^{2}(\theta / 2) \\
& \times\left(\hat{a}+\hat{a}^{+}-\hat{b}-\hat{b}^{+}\right)+\mathrm{i} \hbar \varepsilon\left(\hat{c}^{+}-\hat{c}\right) .
\end{aligned}
$$

Here $\varepsilon=\sqrt{2 \gamma P / \hbar \omega_{L}}$ is related to the input laser power $P, \hat{c}$ and $\hat{c}^{+}$are the annihilation and creation operators, respectively, for the cavity field mode with frequency $\omega_{c}$ and decay rate $\gamma \cdot g=\left(\omega_{c} / L\right) \sqrt{\hbar / m \omega_{m}}$ is optomechanical coupling constant, and $L$ is the cavity length. From this Hamiltonian, quantum Langevin equations (QLEs) [41], which govern the system dynamics, can be derived

$$
\begin{aligned}
& \frac{\mathrm{d} \hat{a}}{\mathrm{~d} t}=-\left(\gamma_{m}+\mathrm{i} \omega_{m}\right) \hat{a}-\frac{\mathrm{i}}{2} G \hat{c}^{+} \hat{c}+\sqrt{2 \gamma_{m}} \hat{a}^{\mathrm{in}}, \\
& \frac{\mathrm{d} \hat{b}}{\mathrm{~d} t}=-\left(\gamma_{m}+\mathrm{i} \omega_{m}\right) \hat{b}+\frac{\mathrm{i}}{2} G \hat{c}^{+} \hat{c}+\sqrt{2 \gamma_{m}} \hat{b}^{\mathrm{in}}, \\
& \frac{\mathrm{d} \hat{c}}{\mathrm{~d} t}=-(\gamma+\mathrm{i} \bar{\Delta}) \hat{c}-\frac{\mathrm{i}}{2} G \hat{c}\left(\hat{a}+\hat{a}^{+}-\hat{b}-\hat{b}^{+}\right) \\
& \quad+\varepsilon+\sqrt{2 \gamma} \hat{c}^{\mathrm{in}},
\end{aligned}
$$

where $G=\sqrt{2} g \cos ^{2}(\theta / 2)$ and $\bar{\Delta}=\omega_{c}-\omega_{L}$ stands for the cavity detuning. $\hat{c}^{\text {in }}$ expresses the quantum noise of the input field. $\hat{a}^{\text {in }}$ and $\hat{b}^{\text {in }}$ are the noise operators for the quantum Brownian motion of the mirrors from the coupling of the movable mirrors to the thermal bath, with mean value zero, and they satisfy

$$
\begin{aligned}
& \left\langle\hat{a}^{\mathrm{in}}(t) \hat{a}^{i n+}\left(t^{\prime}\right)\right\rangle=\left\langle\hat{b}^{\mathrm{in}}(t) \hat{b}^{i n+}\left(t^{\prime}\right)\right\rangle=\left(n_{T}+1\right) \delta\left(t-t^{\prime}\right), \\
& \left\langle\hat{a}^{i n+}(t) \hat{a}^{\mathrm{in}}\left(t^{\prime}\right)\right\rangle=\left\langle\hat{b}^{i n+}(t) \hat{b}^{\mathrm{in}}\left(t^{\prime}\right)\right\rangle=n_{T} \delta\left(t-t^{\prime}\right),
\end{aligned}
$$

where $n_{T}=\left[\exp \left(\hbar \omega_{m} / k_{\mathrm{B}} T\right)-1\right]^{-1}$ is the mean thermal phonon number at equilibrium temperature $T$. $n_{T}$ tends to be zero when $k_{\mathrm{B}} T \ll \hbar \omega_{m}$.

\section{Analysis of steady state}

Stationary solutions have to be analyzed before considering entanglement characteristics since they are related. By setting the time-derivatives to zero in the QLEs and replacing all operators with their expectation values, we get the steady-state equations

$$
\begin{aligned}
& -\left(\gamma_{m}+\mathrm{i} \omega_{m}\right) \alpha-\frac{\mathrm{i}}{2} G\left|c^{s}\right|^{2}=0, \\
& -\left(\gamma_{m}+\mathrm{i} \omega_{m}\right) \beta+\frac{\mathrm{i}}{2} G\left|c^{s}\right|^{2}=0, \\
& -(\gamma+\mathrm{i} \bar{\Delta}) c^{s}-\frac{\mathrm{i}}{2} G c^{s}\left(\alpha+\alpha^{*}-\beta-\beta^{*}\right)+\varepsilon=0 .
\end{aligned}
$$

Here $\alpha, \beta$ are the steady-state amplitudes of the mechanical modes $\hat{a}$ and $\hat{b}$, respectively, and $c^{s}$ is the steady-state amplitude of the intracavity mode $\hat{c}$. Applying the definitions of the quadrature amplitude and phase components $\hat{X}_{m 1}=\hat{a}+\hat{a}^{+}, \hat{Y}_{m 1}=\left(\hat{a}-\hat{a}^{+}\right) / \mathrm{i}$, $\hat{X}_{m 2}=\hat{b}+\hat{b}^{+}, \hat{Y}_{m 2}=\left(\hat{b}-\hat{b}^{+}\right) / \mathrm{i}, \hat{X}=\hat{c}+\hat{c}^{+}$, and $\hat{Y}=\left(\hat{c}-\hat{c}^{+}\right) / i$ and linearization of the operators, we write each operator of quadrature variables of the system as the sum of its steady-state mean value and a small fluctuation $\hat{O}(t)=\bar{O}+\delta \hat{O}(t)$, where $\hat{O}(t)=\left[\hat{X}_{m 1}, \hat{X}_{m 2}, \hat{X}, \hat{Y}_{m 1}, \hat{Y}_{m 2}, \hat{Y}\right]$. We can rewrite the steady-state equations

$$
\begin{aligned}
& \omega_{m} \bar{Y}_{m 1}-\gamma_{m} \bar{X}_{m 1}=0, \quad \omega_{m} \bar{Y}_{m 2}-\gamma_{m} \bar{X}_{m 2}=0, \\
& \bar{\Delta} \bar{Y}-\gamma \bar{X}+\frac{G}{2}\left(\bar{X}_{m 1}-\bar{X}_{m 2}\right) \bar{Y}+2 \varepsilon=0, \\
& \omega_{m} \bar{X}_{m 1}+\gamma_{m} \bar{Y}_{m 1}+G \frac{\bar{X}^{2}+\bar{Y}^{2}}{4}=0, \\
& \omega_{m} \bar{X}_{m 2}+\gamma_{m} \bar{Y}_{m 2}-G \frac{\bar{X}^{2}+\bar{Y}^{2}}{4}=0, \\
& \bar{\Delta} \bar{X}+\gamma \bar{Y}+\frac{G}{2}\left(\bar{X}_{m 1}-\bar{X}_{m 2}\right) \bar{X}=0 .
\end{aligned}
$$

By solving these steady-state equations, we obtain the steady-state mean values

$$
\begin{aligned}
& \bar{X}=\frac{2 \varepsilon \gamma}{\gamma^{2}+\Delta^{2}}, \quad \bar{Y}=-\frac{2 \Delta \varepsilon}{\gamma^{2}+\Delta^{2}} \\
& \Delta=\bar{\Delta}+\frac{G}{2}\left(\bar{X}_{m 1}-\bar{X}_{m 2}\right), \quad \bar{X}_{m 1}=-\bar{X}_{m 2}, \\
& \bar{Y}_{m 1}=\left(\gamma_{m} / \omega_{m}\right) \bar{X}_{m 1}, \quad \bar{Y}_{m 2}=\left(\gamma_{m} / \omega_{m}\right) \bar{X}_{m 2}, \quad \\
& G^{2} \bar{X}_{m 1}^{3}+2 \bar{\Delta} G \bar{X}_{m 1}^{2}+\left(\gamma^{2}+\bar{\Delta}^{2}\right) \bar{X}_{m 1}+\frac{G \omega_{m} \varepsilon^{2}}{\omega_{m}^{2}+\gamma_{m}^{2}}=0 .
\end{aligned}
$$




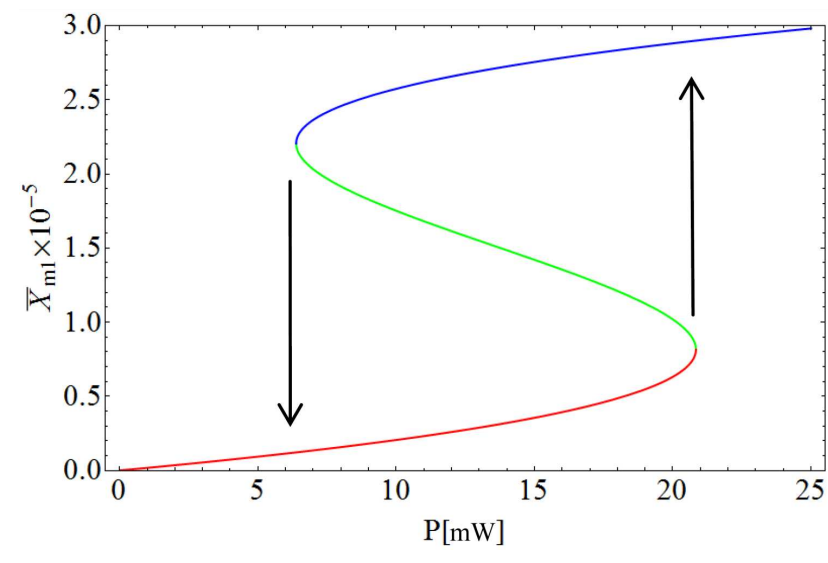

Fig. 2. Bistability of the quadrature amplitude $\bar{X}_{m 1}$ with respect to the input power. The three different color lines correspond to the three solutions, respectively. For the numerical simulations, we use the following experimental parameters: $L=25 \mathrm{~mm}, \theta=\pi / 3$, $\lambda=1064 \mathrm{~nm}, \gamma=2 \pi \times 215 \mathrm{kHz}, m=145 \mathrm{ng}$, $\Delta=-\omega_{m}=2 \pi \times 947 \mathrm{kHz}$ and $\gamma_{m}=2 \pi \times 140 \mathrm{~Hz}$.

The last equation is about the expectation of mechanical quadrature $\bar{X}_{m 1}$, which satisfies a cubic polynomial equation and has three roots. The largest and the smallest roots are stable, and the middle one is unstable. Therefore the system exhibits bistable behavior. Figure 2 shows that the displacement $\bar{X}_{m 1}$ varies with the power of the input field by solving Eqs. (7) numerically, where parameters in the experiment of Ref. [42] are used. These parameters are the ring cavity length $L=25 \mathrm{~mm}$, angle $\theta=\pi / 3$, cavity damping rate $\gamma=2 \pi \times 215 \mathrm{kHz}$ and wavelength of driving laser $\lambda=1064 \mathrm{~nm}$. The resonant frequency, cavity damping rate and effective mass of the mechanical resonators are $\omega_{m}=2 \pi \times 947 \mathrm{kHz}$, $\gamma_{m}=2 \pi \times 140 \mathrm{~Hz}$ and $m=145 \mathrm{ng}$, respectively. It shows that the stability of optomechanical cavity with two identical movable mirrors is similar to that with one movable mirror [43, 44]. This is easy to understand, since the movable mirror changes cavity field through varying cavity length, which is only determined by the differential mode of the two movable mirrors (cf. Eq. (7)). As can be seen from Fig. 2, the bistability can be obtained by scanning the input pump power in two directions. In our case, at about $21 \mathrm{~mW}$, one finds the lower bistable point. The hysteresis then follows the arrow and jumps to the upper branch. To obtain the other unstable point, one needs to scan the input pump power to lower values, which appears at about $6 \mathrm{~mW}$.

Throughout this work, we calculate the simplest case, i.e. input laser power $P<5 \mathrm{~mW}$, where there is only one stationary solution exists (cf. Fig. 2).

\section{Entanglement among mechanical oscillators and cavity reflecting mode}

Now we turn to consider the entanglement of the cavity mode and the two mechanical modes. By introducing quadrature variables operators of the three modes and introducing linearization of the operators, Eqs. (3a)-(3c) can be rewritten as

$$
\begin{aligned}
& \delta \dot{\hat{O}}(t)=M \delta \hat{O}(t)+N \delta \hat{O}^{\mathrm{in}}(t), \\
& \text { with } \\
& \qquad \delta \hat{O}=\left[\delta \hat{X}_{m 1}, \delta \hat{X}_{m 2}, \delta \hat{X}, \delta \hat{Y}_{m 1}, \delta \hat{Y}_{m 2}, \delta \hat{Y}\right]^{\mathrm{T}}, \\
& \qquad \delta \hat{O}^{\mathrm{in}}=\left[\delta \hat{X}_{m 1}^{\mathrm{in}}, \delta \hat{X}_{m 2}^{\mathrm{in}}, \delta \hat{X}^{\mathrm{in}}, \delta \hat{Y}_{m 1}^{\mathrm{in}}, \delta \hat{Y}_{m 2}^{\mathrm{in}}, \delta \hat{Y}^{\mathrm{in}}\right]^{\mathrm{T}},
\end{aligned}
$$

$$
M=\left(\begin{array}{cccccc}
-\gamma_{m} & 0 & 0 & \omega_{m} & 0 & 0 \\
0 & -\gamma_{m} & 0 & 0 & \omega_{m} & 0 \\
0 & 0 & -\gamma & 0 & 0 & \bar{\Delta}-\frac{G^{2} \varepsilon^{2} \omega_{m}}{\gamma^{2}\left(\gamma_{m}^{2}+\omega_{m}^{2}\right)} \\
-\omega_{m} & 0 & -G \varepsilon / \gamma & -\gamma_{m} & 0 & 0 \\
0 & -\omega_{m} & G \varepsilon / \gamma & 0 & -\gamma_{m} & 0 \\
-G \varepsilon / \gamma & G \varepsilon / \gamma & \bar{\Delta}-\frac{G^{2} \varepsilon^{2} \omega_{m}}{\gamma^{2}\left(\gamma_{m}^{2}+\omega_{m}^{2}\right)} & 0 & 0 & -\gamma
\end{array}\right)
$$

and

$$
N=\left(\begin{array}{cccccc}
\sqrt{2 \gamma_{m}} & 0 & 0 & 0 & 0 & 0 \\
0 & \sqrt{2 \gamma_{m}} & 0 & 0 & 0 & 0 \\
0 & 0 & \sqrt{2 \gamma} & 0 & 0 & 0 \\
0 & 0 & 0 & \sqrt{2 \gamma_{m}} & 0 & 0 \\
0 & 0 & 0 & 0 & \sqrt{2 \gamma_{m}} & 0 \\
0 & 0 & 0 & 0 & 0 & \sqrt{2 \gamma}
\end{array}\right) .
$$

By performing the Fourier transform $\hat{O}(\omega)=$ $\int_{-\infty}^{\infty} \mathrm{e}^{-\mathrm{i} \omega t} \hat{O}(t) \mathrm{d} t$ for each operator $\hat{O}$, the equations in the frequency domain may be written as

$$
\delta \hat{O}(\omega)=(-\mathrm{i} \omega E-M)^{-1} N \delta \hat{O}^{\mathrm{in}}(\omega) .
$$

Using the boundary condition $\delta \hat{O}^{\text {out }}(\omega)=\sqrt{2 \gamma} \delta \hat{O}(\omega)-$ $\delta \hat{O}^{\text {in }}(\omega)$, the output amplitude and phase quadratures can be obtained 
$\delta \hat{O}^{\text {out }}(\omega)=\left[N(-\mathrm{i} \omega E-M)^{-1} N-E\right] \delta \hat{O}^{\text {in }}(\omega)$.

Here $E$ is the unit matrix and

$$
\begin{aligned}
& \hat{O}^{\text {out }}(\omega)=\left[\delta \hat{X}_{m 1}^{\text {out }}(\omega), \hat{X}_{m 2}^{\text {out }}(\omega), \hat{X}^{\text {out }}(\omega), \hat{Y}_{m 1}^{\text {out }}(\omega),\right. \\
& \left.\hat{Y}_{m 2}^{\text {out }}(\omega), \hat{Y}^{\text {out }}(\omega)\right] .
\end{aligned}
$$

Then a squeezed laser is applied with squeezing degree $r$ as the input field, whose amplitude fluctuation is $\delta \hat{X}^{\mathrm{in}}(\omega)=\mathrm{e}^{-r}$.

The sufficient inseparability criteria for CV tripartite entanglement is proposed by van Loock and Furusawa [5] and the following three inequalities are given:

$$
\begin{aligned}
& \delta^{2}\left(\hat{Y}_{m 1}^{\text {out }}+\hat{Y}_{m 2}^{\text {out }}\right)+\delta^{2}\left(\hat{X}_{m 1}^{\text {out }}-\hat{X}_{m 2}^{\text {out }}-g_{3} \hat{Y}^{\text {out }}\right)<4, \\
& \delta^{2}\left(\hat{Y}_{m 1}^{\text {out }}+\hat{X}^{\text {out }}\right)+\delta^{2}\left(\hat{X}_{m 1}^{\text {out }}-g_{2} \hat{X}_{m 2}^{\text {out }}-\hat{Y}^{\text {out }}\right)<4, \\
& \delta^{2}\left(\hat{Y}_{m 2}^{\text {out }}-\hat{X}^{\text {out }}\right)+\delta^{2}\left(g_{1} \hat{X}_{m 1}^{\text {out }}-\hat{X}_{m 2}^{\text {out }}-\hat{Y}^{\text {out }}\right)<4,
\end{aligned}
$$

where $g_{1}, g_{2}$, and $g_{3}$ are scaling factors, which are optimized for minimum quantities of the left side. Satisfying these equations is sufficient for full inseparability of GHzlike three-party entanglement, which is class 1 of trimode entanglement defined in Ref. [45]. The smaller the values of the left-hand side of the inequalities are, the larger the correlation degree that we will obtain.

To quantify the degree of the correlation, we introduce the correlation spectra $S_{1}, S_{2}$, and $S_{3}$, which are defined in Eq. (12). Only when all of the values of $S_{1}, S_{2}$, and $S_{3}$ are smaller than 4 , we say that the outgoing field and the two movable mirrors are fully inseparable

$$
\begin{aligned}
& S_{1}=\delta^{2}\left(\hat{Y}_{m 1}^{\text {out }}(\omega)+\hat{Y}_{m 2}^{\text {out }}(\omega)\right)+\delta^{2}\left(\hat{X}_{m 1}^{\text {out }}(\omega)\right. \\
& \left.\quad-\hat{X}_{m 2}^{\text {out }}(\omega)-g_{3} \hat{Y}^{\text {out }}(\omega)\right), \\
& S_{2}=\delta^{2}\left(\hat{Y}_{m 1}^{\text {out }}(\omega)+\hat{X}^{\text {out }}(\omega)\right)+\delta^{2}\left(\hat{X}_{m 1}^{\text {out }}(\omega)\right. \\
& \left.\quad-g_{2} \hat{X}_{m 2}^{\text {out }}(\omega)-\hat{Y}^{\text {out }}(\omega)\right), \\
& S_{3}=\delta^{2}\left(\hat{Y}_{m 2}^{\text {out }}(\omega)-\hat{X}^{\text {out }}(\omega)\right)+\delta^{2}\left(g_{1} \hat{X}_{m 1}^{\text {out }}(\omega)\right. \\
& \left.\quad-\hat{X}_{m 2}^{\text {out }}(\omega)-\hat{Y}^{\text {out }}(\omega)\right) .
\end{aligned}
$$

Figures 3-5 numerically show dependence of the correlation spectra $S_{1}, S_{2}$, and $S_{3}$ on different parameters for $P=2 \mathrm{~mW}$. Figure 3 shows correlation spectra as functions of squeezing parameter $r$ when $n_{T}=0$ and $\Omega=\omega / \gamma=5$. When the injected light is not squeezed, the three parts are entangled. Although, for squeezed light injection, the correlation spectra $S_{2}$ and $S_{3}$ are even smaller. Therefore we claim that squeezing of the injected light enhances the entanglement. It should be noted that $S_{2}=S_{3}$ because of the symmetry of the mirrors $\mathrm{M}_{1}$ and $\mathrm{M}_{2}$, which also applies to Figs. 4 and 5.

Figure 4 shows correlation spectra $S_{1}, S_{2}, S_{3}$ as a function of analysis frequency normalized to cavity bandwidth $\Omega=\omega / \gamma$ with occupation number of mechanical quanta $n_{T}=0$ and squeezing parameter $r=3$. The three parts are entangled when frequency $\Omega \geq 5$.

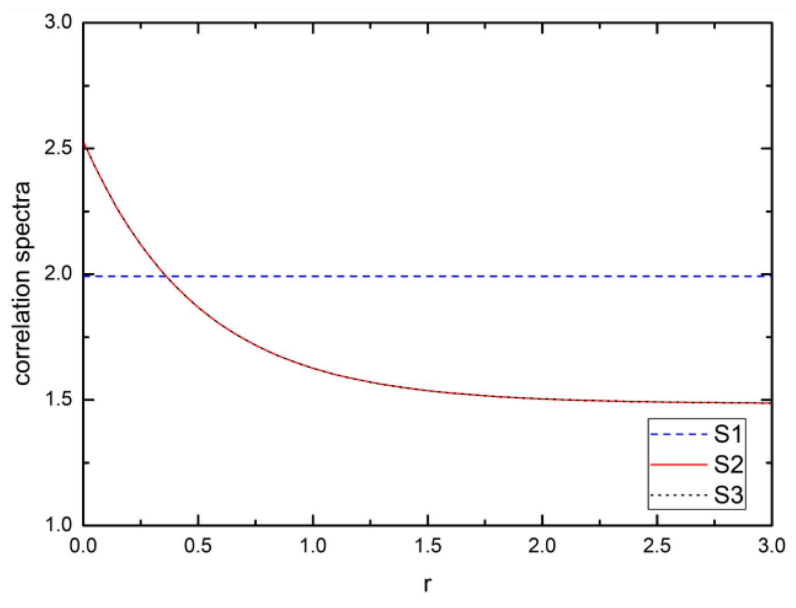

Fig. 3. With $n_{T}=0$, quantum correlation spectra as functions of squeezing degree $r$ at $\Omega=\omega / \gamma=5$.

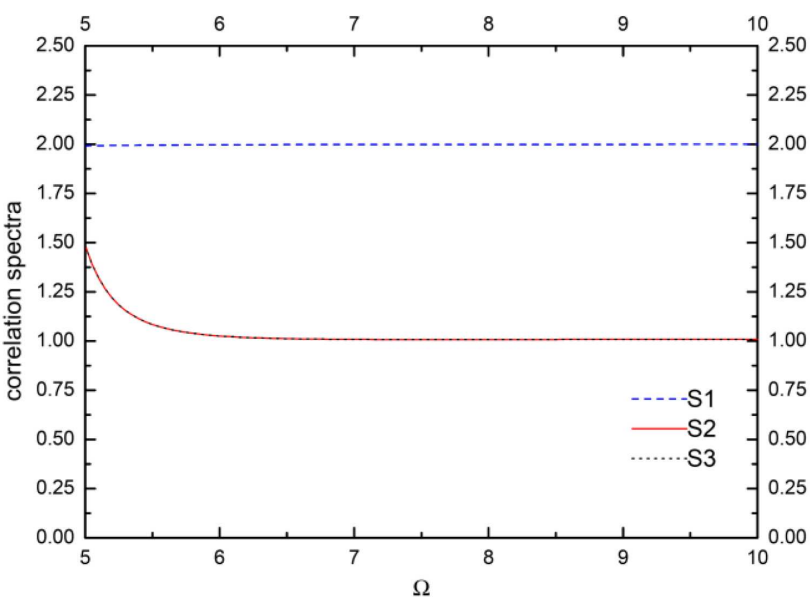

Fig. 4. Quantum correlation spectra versus normalized frequency $\Omega=\omega / \gamma$ with $n_{T}=0$ and $r=3$.

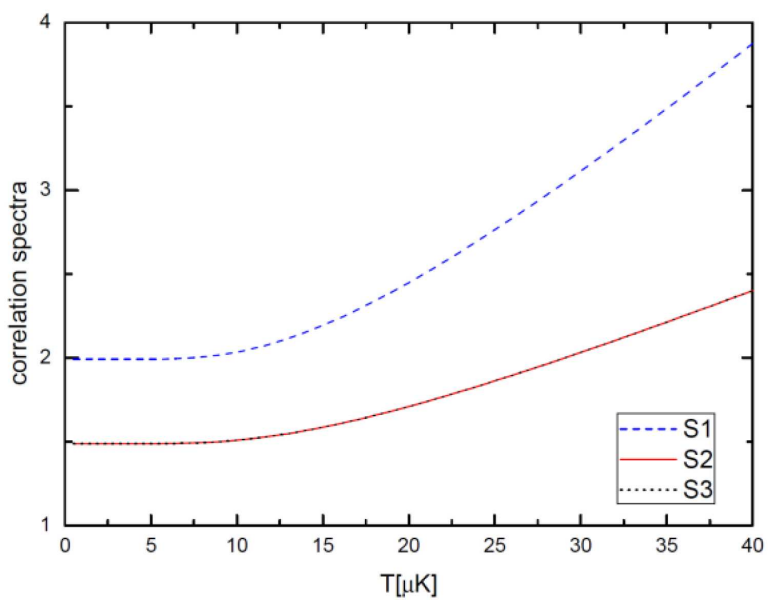

Fig. 5. The influence of temperature $T$ on quantum correlation spectra at $\Omega=5$ and $r=3$. 
The effects of temperature $T$ are shown in Fig. 5, where the Planck formula $n_{T}=\left[\exp \left(\hbar \omega_{m} / k_{\mathrm{B}} T\right)-1\right]^{-1}$ is used. The values of other parameters are $\Omega=5$ and $r=3$. Not surprisingly $S_{1}, S_{2}$, and $S_{3}$ reaches its minimum value at $T=0$, and the three conditions are satisfied when $T \leq$ $40 \mu \mathrm{K}$, which is reachable in state-of-the-art experiment.

\section{Summary}

We have analyzed the tripartite entanglement of an optomechanical ring cavity, which is composed of one fixed partially transmitting mirror and two movable perfectly reflecting mirrors. It is shown that the reflected light from the cavity is entangled with the two movable mirrors when the system is driven by a shot-noise-limited laser. The entanglement can be enhanced if the amplitude noise of the driving laser is squeezed. Fully inseparable tripartite $\mathrm{CV}$ entanglement among the reflected field and the two movable mirrors is generated in a wide range of experimentally accessible parameter regime. Realization of such a scheme will open perspectives for the indirect manipulation of the mirror state through cavityfield control. We expect that these features could find their applications in continuous variable quantum communication networks.

\section{Acknowledgments}

This work is supported in part by National Natural Science Foundation of China (NSFC) (11772177, 91430109, 91536222), Ministry of Science and Technology of the People Republic of China (MOST) (2016YFA0301404) and Shanxi Youth Science and Technology Research Fund (201701D12111501).

\section{References}

[1] D. Bouweester, A. Ekert, A. Zeilinger, The Physics of Quantum Information, Springer-Verlag, Berlin 2000.

[2] M.A. Nielsen, I.L. Chuang, Quantum Computation and Quantum Information, Cambridge University Press, Cambridge 2011.

[3] H.J. Kimble, Nature 453, 1023 (2008).

[4] T. Aoki, N. Takei, H. Yonezawa, K. Wakui, T. Hiraoka, A. Furusawa, Phys. Rev. Lett. 91, 080404 (2003).

[5] P.V. Loock, A. Furusawa, Phys. Rev. A 67, 052315 (2002).

[6] A.S. Coelho, F.A.S. Barbosa, K.N. Cassemiro, A.S. Villar, M. Martinelli, P. Nussenzveig, Science 326, 823 (2009).

[7] X. Su, A. Tan, X. Jia, J. Zhang, C. Xie, K. Peng, Phys. Rev. Lett. 98, 070502 (2007).

[8] P.V. Loock, S.L. Braunstein, Phys. Rev. A 63, 022106 (2001).

[9] M. Yukawa, R. Ukai, P.V. Loock, A. Furusawa, Phys. Rev. A 78, 012301 (2008).

[10] Y.B. Yu, Z.D. Xie, X.Q. Yu, H.X. Li, P. Xu, H.M. Yao, S.N. Zhu, Phys. Rev. A 74, 042332 (2006).
[11] J. Guo, H. Zou, Z. Zhai, J. Zhang, J. Gao, Phys. Rev. A 71, 034305 (2005).

[12] J. Guo, Z. Zhai, J. Gao, J. Opt. Soc. Am. B 27, 518 (2010).

[13] J. Guo, S. Zhai, Z. Zhai, Ann. Phys. 27, 814 (2016).

[14] H. Yonezawa, T. Aoki, A. Furusawa, Nature 431, 430 (2004).

[15] J. Jing, J. Zhang, Y. Yan, F. Zhao, C. Xie, K. Peng, Phys. Rev. Lett. 90, 167903 (2003).

[16] H.K. Lau, C. Weedbrook, Phys. Rev. A 88, 042313 (2013).

[17] H.J. Briegel, R. Raussendorf, Phys. Rev. Lett. 86, 910 (2001).

[18] S. Yokoyama, R. Ukai, S.C. Armstrong, J.I. Yoshikawa, P.V. Loock, A. Furusawa, Phys. Rev. A 92, 032304 (2015).

[19] J.K. Kalaga, W. Leoński, J. Peřina, Jr., Phys. Rev. A 97, 042110 (2018).

[20] R.S. Said, M.R.B. Wahiddin, B.A. Umarov, J. Phys. B At. Mol. Opt. Phys. 39, 1269 (2006).

[21] J.K. Kalaga, W. Leonski, R. Szczesniak, Quantum Inf. Process. 16, 265 (2017).

[22] J.K. Kalaga, A. Kowalewska-Kudłaszyk, W. Leonski, A. Barasinski, Phys. Rev. A 94, 032304 (2016).

[23] M.K. Olsen, Phys. Rev. A 92, 033627 (2015).

[24] D. Vitali, S. Gigan, A. Ferreira, H.R. Böhm, P. Tombesi, A. Guerreiro, V. Vedral, A. Zeilinger, M. Aspelmeyer, Phys. Rev. Lett. 98, 030405 (2007).

[25] C. Genes, A. Mari, P. Tombesi, D. Vitali, Phys. Rev. A 78, 032316 (2008).

[26] Y.H. Ma, L. Zhou, J. Appl. Phys. 111, 103109 (2012).

[27] Z. Dan, Z.X. Ping, Z. Qiang, Chin. Phys. B 22, 064206 (2013).

[28] J. Zhang, K. Peng, S.L. Braunstein, Phys. Rev. A 68, 013808 (2003).

[29] S. Mancini, V. Giovannetti, D. Vitali, P. Tombesi, Phys. Rev. Lett. 88, 120401 (2002).

[30] M. Pinard, A. Dantan, D. Vitali, O. Arcizet, T. Briant, A. Heidmann, Europhys. Lett. 72, 747 (2005).

[31] D. Vitali, S. Mancini, Tombesi, J. Phys. A Math. Theor. 40, 8055 (2007).

[32] S. Huang, G.S. Agarwal, New J. Phys. 11, 103044 (2009).

[33] C. Joshi, J. Larson, M. Jonson, E. Andersson, P. Öhberg, Phys. Rev. A 85, 033805 (2012).

[34] H. Tan, L.F. Buchmann, H. Seok, G. Li, Phys. Rev. A 87, 022318 (2013).

[35] G. Vacanti, M. Paternostro, G.M. Palma, V. Vedral, New J. Phys. 10, 095014 (2008).

[36] X. Yang, Y. Ling, X. Shao, M. Xiao, Phys. Rev. A 95, 052303 (2017).

[37] M. Paternostro, D. Vitali, S. Gigan, M.S. Kim, C. Brukner, J. Eisert, M. Aspelmeyer, Phys. Rev. Lett. 99, 250401 (2007).

[38] S. Pirandola, S. Mancini, D. Vitali, P. Tombesi, Phys. Rev. A 68, 062317 (2003). 
[39] H. Miao, C. Zhao, L. Ju, D.G. Blair, Phys. Rev. A 79, 063801 (2009).

[40] Y.D. Wang, S. Chesi, A.A. Clerk, Phys. Rev. A 91, 013807 (2015).

[41] M.J. Collett, C.W. Gardiner, Phys. Rev. A 30, 1386 (1984).

[42] S. Gröblacher, K. Hammerer, M.R. Vanner, M. Aspelmeyer, Nature 460, 724 (2009).
[43] E.A. Sete, H. Eleuch, Phys. Rev. A 85, 043824 (2012).

[44] H. Xiong, L.G. Si, A.S. Zheng, X. Yang, Y. Wu, Phys. Rev. A 86, 013815 (2012).

[45] G. Giedke, B. Kraus, M. Lewenstein, J.I. Cirac, Phys. Rev. A 64, 052303 (2001). 